\title{
Impact of Osmotica and Abscisic Acid on Direct Somatic Embryogenesis in Tea
}

\author{
M. Suganthi ${ }^{1}$, S. Arvinth ${ }^{1,2}$, R. Raj Kumar ${ }^{1, *}$ \\ ${ }^{1}$ Plant Physiology and Biotechnology Division, UPASI Tea Research Institute, Valparai, Coimbatore District 642127, Tamil Nadu, India \\ ${ }^{2}$ Department of Crop Improvement, Vanavarayar Institute of Agriculture, Pollachi - 642103, Tamil Nadu, India
}

\begin{abstract}
An in vitro protocol was developed to induce somatic embryos using mature cotyledons obtained from open pollinated flowers of cultivated Camellia species. Impact of abscisic acid (ABA) alone and its combination with osmotica was also evaluated on somatic embryogenesis. Various concentrations of osmoticum viz., polyethylene glycol (PEG) or mannitol or glycine betaine were tested where ABA $5 \mathrm{mg} \mathrm{L}-1$ and PEG 3\% increased the number of somatic embryo formation compared to the other osmoticum coupled with ABA. Results suggested that the use of osmoticum along with ABA was quite effective to induce somatic embryos in tea. But germination of embryos required a new combination of nutrient medium. Inclusion of 6-benzylaminopurine (BAP) and gibberellic acid (GA3) in the nutrient medium enhanced percent germination and its growth.
\end{abstract}

Keywords Tea cotyledons, PEG, Glycine betaine, Mannitol

\section{Introduction}

Tea is an important socio-economic crop and the highest consumed non-alcoholic beverage in the world. It is proven that bio-molecules like flavonoids and antioxidants in tea possess medicinal properties ${ }^{[1]}$. Reports on tea tissue culture including somatic embryogenesis are available ${ }^{[2-8]}$. However, rapid multiplication is very poor as the plant is relatively recalcitrant. Moreover, vegetative propagation technology is fine tuned and cost effective as well. On the other hand, somatic embryogenesis is attempted till date in order to refine a trait specific accession(s) or accelerate the research on in vitro breeding. Tea clones are characterized as tolerant/susceptible to stress; but they have not been effectively exploited under in vitro breeding programmes to refine the existing genetic make up.

In earlier tea tissue culture studies, either ABA or osmoticum was not reported with particular reference to somatic embryogenesis or resistance induction ${ }^{[9]}$. Generally osmoticum has the capacity to induce water stress which in turn responsible for somatic embryo formation and to induce stress tolerance. PEG is a non-plosmolysing osmoticum which enhances the somatic embryo maturation by increasing osmolality of the culture medium ${ }^{[10]}$. ABA is one of the five classical plant growth regulating substances which involved in the somatic embryo induction. It has already been

* Corresponding author:

weganr@rediffmail.com (R. Raj Kumar)

Published online at http://journal.sapub.org/plant

Copyright (C) 2012 Scientific \& Academic Publishing. All Rights Reserved established that combination of ABA and osmoticum on somatic embryogenesis of many crop plants ${ }^{[11]}$. On the above grounds, as a first step in in vitro breeding for refinement of trait specific cultivar an attempt has been made. The present study was also deals with elucidation of impact of ABA and its combination with various osmotica on somatic embryo induction and maturation besides somatic embryo germination in tea.

\section{Materials and Methods}

\subsection{Plant Material and Explant Preparation}

Mature fruits obtained through open pollination representing "China" hybrids, UPASI-9, UPASI-10 \& ATK-1 and "Cambod" variety UPASI-17 were collected from UPASI Experimental Farm and used as explants. Seeds were separated from the field collected fruits and were kept under running tap water to remove the floaters. Seeds were thoroughly washed with Teepol for 3 minutes followed by 3 rinses with sterile distilled water. Subsequently seeds were surface sterilized with $0.1 \%$ mercuric chloride for $20 \mathrm{~min}$ utes followed by 3 rinses with sterile distilled water for $5 \mathrm{~min}$, under aseptic conditions. At the time of culturing, seed coat was removed mechanically, zygotic embryo detached from its axis and mature cotyledons without embryo cut into pieces were used as explants in the present study.

\subsection{Culture Medium and Conditions}

Excised cotyledons were cultured on basal MS medium ${ }^{[12]}$ containing $200 \mathrm{mg} / \mathrm{L}$ of L-glutamine as an additional organic 
nitrogen source ${ }^{[13]}$. After 3-4 weeks of culturing, globular shape, tiny, somatic embryos were emerged from the surface of the cotyledons. While sub-culturing, they were transferred on medium containing various concentrations of $\mathrm{ABA}$ (0.5-6.0mg/L) to document the impact of ABA on somatic embryogenesis. On somatic embryo maturation, globular shaped somatic embryos were cultured on MS medium supplemented with ABA $(5 \mathrm{mg} / \mathrm{L})$ and different concentrations of osmoticum, (i) PEG $(5.0-50 \mathrm{~g} / \mathrm{L})$ or (ii) mannitol $(5.0-50 \mathrm{~g} / \mathrm{L})$ or (iii) glycine betaine $(100-1000 \mathrm{mg} / \mathrm{L})$. Since, prolonged culture on ABA containing medium did not favour germination, after 4 weeks, physiologically uniform matured somatic embryos were transferred on to germination medium containing $5.0 \mathrm{mg} / \mathrm{L} \mathrm{GA}_{3}$ and $2 \mathrm{mg} / \mathrm{L}$ 6- benzylaminopurine (BAP). For shoot elongation, these cultures were transferred onto MS medium supplemented with $3.0 \mathrm{mg} / \mathrm{L} \mathrm{GA}_{3}$. Extended period of incubation on shoot induction and elongation media did not accelerate the root growth where the radical become enlarged and its growth was stunted. On elongation, shoots were transferred onto MS medium containing $5.0 \mathrm{mg} \mathrm{L}^{-1}$ indole-3-butyric acid (IBA) for rapid root growth. In all the cases, $\mathrm{pH}$ of the culture media was adjusted to $5.7-5.8$ prior to addition of agar $(0.7 \mathrm{~g}$ $\mathrm{L}^{-1}$ ) and sterilized at $121^{\circ} \mathrm{C}$ for 20 minutes.

All cultures were incubated under light (16:8 h photoperiod) at $24 \pm 2^{\circ} \mathrm{C}$ for somatic embryogenesis. Cultures were sub-cultured at 4 week interval on the same medium concentration, unless otherwise specified.

\subsection{Statistical Analysis}

Each experiment was repeated three times with 25 replicates (or cultures). Periodical observations were recorded on somatic embryo formation and maturation. Data generated were subjected to analysis of variance (ANOVA).

\section{Results and Discussion}

\subsection{Impact of ABA on Direct Somatic Embryogenesis}

Considering the clones, except UPASI-17, all other three belongs to "China" hybrids which highly resist soil moisture stress. On the other hand, UPASI-17 represent broad leaved "Cambod" variety fairly thrives against stress. In south Indian tea plantations, UPASI-9 and UPASI-17 were predominantly used as planting material in replanting programme. In the present study, among the clones selected UPASI-9 and UPASI-10 highly responded under culture conditions where the MS basal medium augmented with ABA followed by UPASI-17 (Table 1). However, response of ATK-1 was on bar with UPASI-17 under culture conditions irrespective of the ABA concentrations. Value of co-efficient of variation was $>15 \%$ which may be attributed to the clonal variation and other factors like explant sampling time.

Heart shaped somatic embryos was observed during maturation stage on MS medium containing ABA (5.0 mg
$\mathrm{L}^{-1}$ ) (Figure 1b.). When the ABA concentration increases from 0.5 to $5.0 \mathrm{mg} \mathrm{L}^{-1}$, there was a linear increase in somatic embryo induction. ABA beyond $5.5 \mathrm{mg} \mathrm{L}^{-1}$ reduces the number of somatic embryos per culture. There had been a quadratic relationship existed between ABA concentration and number of somatic embryos fitting the exponential model $\left(y=a+b x+c x^{2}\right)$ where the correlation co-efficient value was highly significant at $1 \%$ level $\left(r^{2}=0.951\right)$. On the basis of numbers of somatic embryos produced, medium containing $5.0 \mathrm{mg} \mathrm{L}^{-1}$ of $\mathrm{ABA}$ was taken up for further studies (Table 1).

Even though, ABA alone found to induce somatic embryos and their multiplication, as it impart dormancy, no shoot growth was noticed from the somatic embryos. Even after transferring the matured somatic embryos from ABA containing medium to shoot induction medium, it took more than 2 months for shoot induction. It is quite obvious that the residual effect of incorporation of ABA in the medium suppressed the shoot induction. However, root induction was evident from somatic embryos when cultured on ABA containing medium for longer duration (Figure1c.). These cultures were maintained over a period of 5 months by frequent sub-culturing but no shoot induction was observed. ABA alone shows no induction stimulus in explants, rather than its inhibitory action. Shoot induction response was enhanced only when ABA was supplemented with stress factor betaine $^{[14]}$. Due to the inhibitory action of ABA, in many plants $\mathrm{ABA}$ was used along with osmotica or in combination with other hormones. In neem, ABA was used along with thidiazuron for induction of somatic embryos ${ }^{[15]}$.

\subsection{Effect of PEG Along with ABA on Direct Somatic Embryogenesis}

$\mathrm{ABA}$ and PEG combination were frequently used for somatic embryo maturation in many plant species like Horse chestnet $^{[16]}$, conifer ${ }^{[17]}$, Medicago ${ }^{[18]}$, Panax ginseng ${ }^{[19]}$, Hevea brasiliensis ${ }^{[20]}$ and in white spruce ${ }^{[21]}$. On the other hand, effect of PEG along with ABA on somatic embryogenesis has not been reported in tea before. As mentioned earlier, globular shaped somatic embryos formed on glutamine containing medium were transferred onto various concentrations of osmoticum along with $\mathrm{ABA}$ to monitor the effect of somatic embryo maturation and plant development. Somatic embryo maturation varied when MS medium augmented with 0.5-5.0 $\mathrm{g} \mathrm{L}^{-1}$ PEG along with constant concentration of $\mathrm{ABA}\left(5.0 \mathrm{mg} \mathrm{L}^{-1}\right)$. But clones did not exhibit any variation in terms of somatic embryo formation and maturation (Table 2). In certain cases, interaction between clones and osmoticum + ABA concentration was found significant. There was a quadratic relationship existed between $\mathrm{ABA}+$ PEG concentration and induction of number of somatic embryos. The correlation co-efficient value is highly significant at $1 \%$ level $\left(r^{2}=0.850\right)$. Number of somatic embryos produced on PEG $\left(30 \mathrm{~g} \mathrm{~L}^{-1}\right)$ containing medium was as high as 20 (Figure 1d.) and higher concentration of PEG along with $\mathrm{ABA}$ inhibited the somatic embryo production. Thirty 
days after culture, about $50 \%$ of somatic embryos germinated on shoot induction medium where the BAP nullified the residual effect of ABA. Germination of somatic embryos may also be attributed to the synthesis of storage protein and its effect on precocious germination as it was observed in white spruce ${ }^{[22]}$.

\subsection{Efficacy of Mannitol and Glycine Betaine on Direct Somatic Embryogenesis}

There was no significant variation in somatic embryo formation among the clones, irrespective of $\mathrm{ABA}$ and mannitol concentration. Considering the $\mathrm{ABA}$ and mannitol concentration individually, somatic embryo induction was higher when the cotyledons cultured on the medium containing ABA $5.0 \mathrm{mg} \mathrm{L}^{-1}+$ mannitol $25 \mathrm{~g} \mathrm{~L}^{-1}$ (Table 3). Unlike the effect of PEG and mannitol, increasing level of glycine betaine did not show identical trend in terms of somatic embryo production and maturation (Table 4). Among the clones studied, UPASI-17 exhibited poor response in inducing number of somatic embryos on glycine betaine containing medium. Compared to PEG, the mean number of somatic embryos obtained on MS medium augmented with mannitol $(0.5-5.0 \mathrm{~g} / \mathrm{L})$ or glycine betaine $\left(100-1000 \mathrm{mg} \mathrm{L}^{-1}\right)$ was low (Tables 2-4).

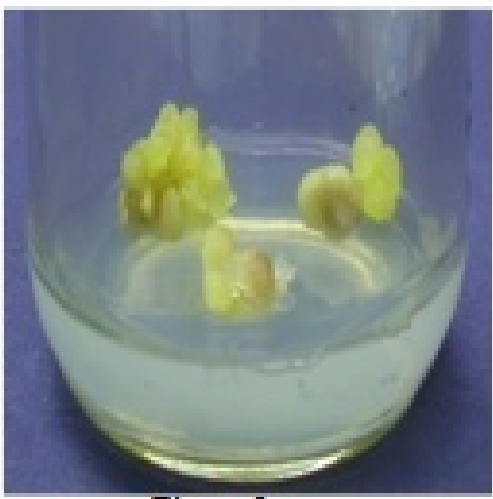

Figure la

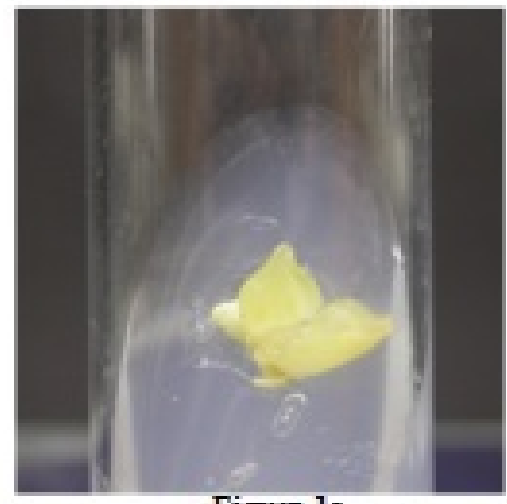

Figure lc.

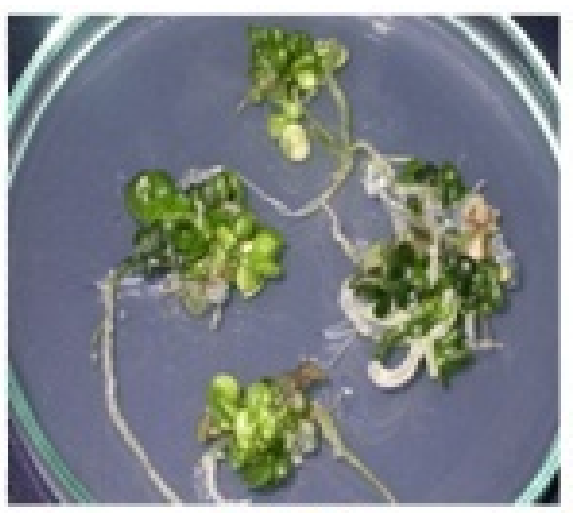

Figure lc

\section{Within 4-6 weeks of culturing on shoot induction medium,} germination of somatic embryos was observed (Figure 1e.). Since the plants obtained were tiny with rosette nodes, these were transferred onto shoot elongation medium. As mentioned earlier, radical was swollen and thick on shoot induction and elongation media. In order to accelerate the rapid growth of the root initial, these cultures were transferred on to medium containing $5.0 \mathrm{mg} \mathrm{L}^{-1} \mathrm{IBA}$ where proliferation of roots was observed in all cultures.

Irrespective of the somatic embryo derived from the ABA + various concentrations of osmotica and cultivar types, they were cultured on germination medium. Per cent germination of somatic embryo varied between 11 and 51, irrespective of the clones (Table 5). Residual effect of higher concentration of osmotica was observed in terms of per cent germination of somatic embryos where the higher concentration of osmoticum may be responsible for low germination potential. A similar low plant conversion through somatic embryogenesis was reported in vitis latifolia ${ }^{[23]}$.

Results suggest that ABA, when used alone inhibit germination potential of somatic embryos but in combination with osmoticum accelerated multiplication and maturation of the same.

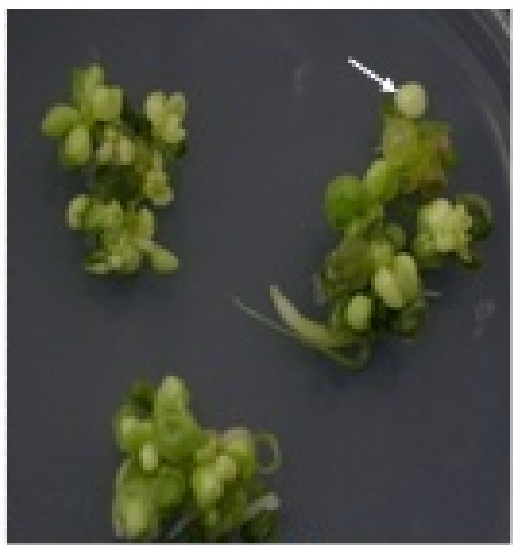

Figure ld.

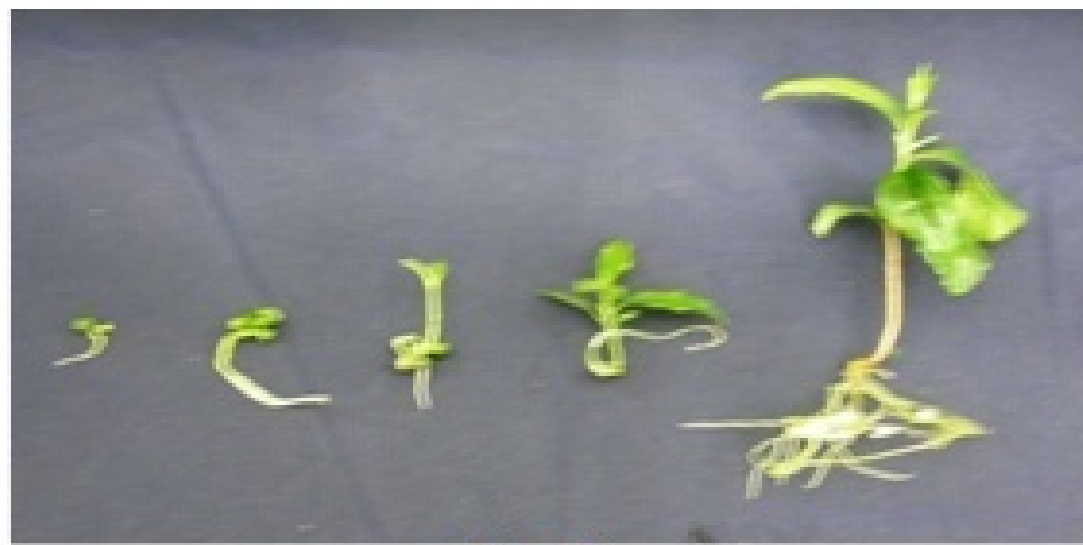

Figure le.

Figure 1a-e. Different stages of direct somatic embryogenesis in tea:(a) Globular shaped somatic embryos directly regenerated from the cotyledons, (b) Heart-shaped somatic embryos, (c) Root development when treated with ABA alone without any shoot induction, (d) Effect of 3 g L-1 PEG and 5 mg L-1 ABA on multiplication of somatic embryos (arrows indicate globular embryo), (e) Different developmental stages of somatic embryo germination 
Table 1. Clonal Variation and Impact of ABA Concentration on Somatic Embryo Induction in Tea

\begin{tabular}{|c|c|c|c|c|c|}
\hline \multicolumn{6}{|c|}{ Cultivar } \\
\hline $\mathrm{ABA}(\mathrm{mg} / \mathrm{L})$ & UPASI-9 & UPASI-10 & UPASI-17 & ATK-1 & Mean Concentration \\
\hline 0.5 & 2.7 & 3.0 & 3.0 & 2.0 & 2.7 \\
\hline 1.0 & 2.7 & 4.7 & 4.7 & 3.0 & 3.8 \\
\hline 2.0 & 4.7 & 5.0 & 5.0 & 2.7 & 4.3 \\
\hline 2.5 & 7.0 & 9.3 & 6.0 & 10.3 & 8.2 \\
\hline 3.0 & 16.3 & 12.3 & 9.0 & 9.0 & 11.7 \\
\hline 4.0 & 19.0 & 15.0 & 11.0 & 11.0 & 14.0 \\
\hline 4.5 & 17.3 & 16.3 & 12.7 & 11.0 & 14.3 \\
\hline 5.0 & 19.3 & 17.3 & 16.3 & 18.3 & 17.8 \\
\hline 5.5 & 16.3 & 19.7 & 13.0 & 9.0 & 14.5 \\
\hline 6.0 & 17.0 & 19.0 & 12.7 & 10.7 & 14.8 \\
\hline Mean Cultivar & 12.2 & 12.2 & 9.3 & 8.7 & \\
\hline \multicolumn{6}{|c|}{ Statistical significance at $\mathrm{P}=0.05$ : } \\
\hline & \multicolumn{2}{|c|}{$\mathrm{SE}^{*}$} & \multicolumn{2}{|c|}{ C.D. } & C.V. (\%) \\
\hline PGR Conc (T) & \multicolumn{2}{|c|}{0.74} & \multicolumn{2}{|c|}{1.45} & 17.1 \\
\hline Cultivar (C) & \multicolumn{2}{|c|}{0.47} & \multicolumn{2}{|c|}{0.92} & \\
\hline Interaction $(\mathrm{T} \times \mathrm{C})$ & \multicolumn{2}{|c|}{1.48} & \multicolumn{2}{|c|}{2.91} & \\
\hline
\end{tabular}

Table 2. Interaction Between PEG and ABA on Somatic Embryogenesis in Tea

\begin{tabular}{|c|c|c|c|c|c|}
\hline $\mathrm{ABA}(5 \mathrm{mg} / \mathrm{L})+$ & \multicolumn{4}{|c|}{ Cultivar } & \multirow[b]{2}{*}{ Mean Concentration } \\
\hline PEG (g/L) & UPASI-9 & UPASI-10 & UPASI-17 & ATK-1 & \\
\hline 5 & 7.0 & 8.0 & 5.3 & 7.3 & 6.9 \\
\hline 10 & 8.0 & 10.3 & 6.0 & 8.0 & 8.1 \\
\hline 15 & 8.3 & 8.3 & 7.0 & 8.0 & 7.9 \\
\hline 20 & 10.3 & 12.3 & 12.0 & 10.3 & 11.3 \\
\hline 25 & 14.7 & 18.3 & 17.0 & 17.0 & 16.8 \\
\hline 30 & 20.3 & 17.7 & 21.3 & 20.3 & 19.9 \\
\hline 35 & 14.0 & 13.0 & 15.7 & 14.3 & 14.3 \\
\hline 40 & 9.3 & 10.0 & 7.3 & 8.3 & 8.8 \\
\hline 45 & 4.0 & 5.7 & 2.7 & 4.3 & 4.2 \\
\hline 50 & 3.7 & 2.7 & 3.0 & 2.3 & 2.9 \\
\hline Mean (Cultivar) & 10.0 & 10.6 & 9.7 & 10.0 & \\
\hline \multicolumn{6}{|c|}{ Statistical significance at $\mathrm{P}=0.05$ : } \\
\hline & \multicolumn{2}{|c|}{$\mathrm{SE}^{*}$} & \multicolumn{2}{|c|}{ C.D. } & C.V. $(\%)$ \\
\hline PGR Conc (T) & \multicolumn{2}{|c|}{0.73} & \multicolumn{2}{|c|}{1.44} & 17.8 \\
\hline Cultivar (C) & \multicolumn{2}{|c|}{0.46} & \multicolumn{2}{|c|}{0.91} & \\
\hline Interaction $(\mathrm{T} \times \mathrm{C})$ & \multicolumn{2}{|c|}{1.47} & \multicolumn{2}{|c|}{2.88} & \\
\hline
\end{tabular}

Table 3. Impact of Mannitol in Combination with ABA on Somatic Embryogenesis in Tea

\begin{tabular}{|c|c|c|c|c|c|}
\hline $\mathrm{ABA}(5 \mathrm{mg} / \mathrm{L})+$ & \multicolumn{4}{|c|}{ Cultivar } & \multirow[b]{2}{*}{ Mean Concentration } \\
\hline Mannitol (g/L) & UPASI-9 & UPASI-10 & UPASI-17 & ATK-1 & \\
\hline 5 & 1.3 & 3.7 & 2.7 & 3.3 & 2.8 \\
\hline 10 & 1.3 & 5.3 & 3.7 & 4.3 & 3.7 \\
\hline 15 & 7.3 & 7.3 & 10.3 & 7.7 & 8.2 \\
\hline 20 & 8.0 & 9.3 & 11.7 & 8.3 & 9.3 \\
\hline 25 & 9.3 & 11.0 & 12.7 & 11.3 & 11.1 \\
\hline 30 & 7.7 & 6.0 & 9.3 & 7.7 & 7.7 \\
\hline 35 & 9.3 & 12.0 & 7.7 & 9.3 & 9.6 \\
\hline 40 & 7.7 & 8.7 & 3.7 & 6.3 & 6.6 \\
\hline 45 & 8.7 & 9.3 & 8.0 & 8.0 & 8.5 \\
\hline 50 & 4.0 & 6.0 & 7.3 & 6.0 & 5.8 \\
\hline Mean (Cultivar) & 6.5 & 7.9 & 7.7 & 7.2 & \\
\hline \multicolumn{6}{|c|}{ Statistical significance at $\mathrm{P}=0.05$ : } \\
\hline & \multicolumn{2}{|c|}{$\mathrm{SE}^{*}$} & \multicolumn{2}{|c|}{ C.D. } & C.V. $(\%)$ \\
\hline PGR Conc (T) & \multicolumn{2}{|c|}{0.92} & \multicolumn{2}{|c|}{1.80} & 13.7 \\
\hline Cultivar (C) & \multicolumn{2}{|c|}{0.58} & \multicolumn{2}{|c|}{1.14} & \\
\hline Interaction $(\mathrm{T} \times \mathrm{C})$ & \multicolumn{2}{|c|}{1.84} & \multicolumn{2}{|c|}{3.60} & \\
\hline
\end{tabular}

$\mathrm{SE}^{*}:$ standard error mean; C.D.: critical difference at 5\% probability and C.V.(\%): co-efficient of variation 
Table 4. Effect of Various Concentrations of Glycine betaine and ABA on Somatic Embryogenesis in Tea

\begin{tabular}{|c|c|c|c|c|c|}
\hline $\mathrm{ABA}(5 \mathrm{mg} / \mathrm{L})+$ & \multicolumn{4}{|c|}{ Cultivar } & \multirow[b]{2}{*}{ Mean Concentration } \\
\hline Glycine betaine $(\mathrm{mg} / \mathrm{L})$ & UPASI-9 & UPASI-10 & UPASI-17 & ATK-1 & \\
\hline 100 & 10.7 & 7.3 & 4.3 & 6.3 & 7.2 \\
\hline 200 & 8.0 & 4.3 & 6.3 & 6.3 & 6.3 \\
\hline 300 & 9.3 & 6.3 & 5.7 & 6.7 & 7.0 \\
\hline 400 & 17.3 & 14.0 & 10.7 & 13.7 & 13.9 \\
\hline 500 & 11.0 & 10.3 & 6.3 & 8.3 & 8.9 \\
\hline 600 & 15.7 & 8.0 & 6.3 & 10.3 & 10.1 \\
\hline 700 & 15.0 & 4.3 & 5.3 & 9.3 & 8.5 \\
\hline 800 & 14.7 & 21.3 & 8.0 & 14.7 & 14.7 \\
\hline 900 & 12.0 & 16.3 & 2.3 & 15.0 & 11.4 \\
\hline 1000 & 10.3 & 11.7 & 3.7 & 10.0 & 8.9 \\
\hline Mean (Cultivar) & 12.4 & 10.4 & 5.9 & 10.0 & \\
\hline \multicolumn{6}{|c|}{ Statistical significance at $\mathrm{P}=0.05$ : } \\
\hline & \multicolumn{2}{|c|}{$\mathrm{SE}^{*}$} & \multicolumn{2}{|c|}{ C.D. } & C.V. $(\%)$ \\
\hline PGR Conc (T) & \multicolumn{2}{|c|}{0.78} & \multicolumn{2}{|c|}{1.53} & 9.7 \\
\hline Cultivar (C) & \multicolumn{2}{|c|}{0.49} & \multirow{2}{*}{\multicolumn{2}{|c|}{0.97}} & \\
\hline Interaction $(\mathrm{T} \times \mathrm{C})$ & \multicolumn{2}{|c|}{1.56} & & 3.05 & \\
\hline
\end{tabular}

Table 5. Germination Response of Different Osmotica Treated Somatic Embryos

\begin{tabular}{cccccc}
\hline $\begin{array}{c}\text { PEG } \\
(\mathrm{g} / \mathrm{L})\end{array}$ & Germination (\%) & $\begin{array}{c}\text { Mannitol } \\
(\mathrm{g} / \mathrm{L})\end{array}$ & Germination $(\%)$ & $\begin{array}{c}\text { Glycine betaine } \\
(\mathrm{g} / \mathrm{L})\end{array}$ & Germination $(\%)$ \\
5 & 11.1 & 5 & 30 & 100 & 14.2 \\
10 & 18.7 & 10 & 23 & 200 & 16.6 \\
15 & 25.8 & 15 & 29 & 300 & 17.8 \\
20 & 37.7 & 20 & 18.9 & 400 & 18.8 \\
25 & 24.2 & 25 & 38.6 & 500 & 20 \\
30 & 51.2 & 30 & 26.6 & 600 & 20 \\
35 & 41 & 35 & 21 & 700 & 33.3 \\
40 & 29.4 & 40 & 11.5 & 800 & 42 \\
45 & 23.5 & 45 & 11.7 & 900 & 40.8 \\
50 & 30 & 50 & 11.1 & 1000 & 37.5 \\
\hline
\end{tabular}

\section{Conclusions}

In nut shell, a new, simple and repeatable methodology was standardized for direct somatic embryogenesis in tea. PEG and ABA induced considerably higher number of somatic embryos. This protocol can be adopted for in vitro plant improvement programmes using trait specific cultivars and its further improvement. In recent years, somatic embryos are being frequently used in genetic transformation studies, results obtained in the present study will also serve as a rapid source of explant.

\section{ACKNOWLEDGEMENTS}

Authors are thankful to Dr. P. Mohankumar, Director, UPASI Tea Research Institute for his encouragement and support.

\section{REFERENCES}

[1] Ghanati, F., and Ragmati ishka, M., 2009, Investigation of the interaction between abscisic acid (ABA) and excess benzyladenine (BA) on the formation of shoot in tissue culture of tea (Camellia sinensis L.)., In J. Plant Prod., 3(4), 735-8043.
[2] Tahardi, J. S., Imron Riadi, Dodd, W. A., 2003, Enhancement of somatic embryo development and plantlet recovery in Camellia sinensis by temporary liquid immersion., J. Biotek. Pertanian., 8(1), 1-7.

[3] Mariya John, K. M., and Raj Kumar, R., 2006, Factors influencing synthetic seed germination in tea (Camellia sinensis (L.) O. Kuntze)., J. Plantn. Crops., 34(1), 40-42.

[4] Furukawa, K., and Tanaka, J., 2004, 'Makura-Ck2': A tea strain with a high somatic embryogenesis., Breed. Res., 6(3), 109-115.

[5] Mondal, T. K., Bhattacharya, A., Ahuja, P. S., 2001a, Induction of synchronous secondary somatic embryogenesis in Camellia sinensis (L.) O. Kuntze., J. Plant Physiol., 158(7), 945-951.

[6] Akula, A., Becker, D., Bateson, M., 2000a, High-yielding repetitive somatic embryogenesis and plant recovery in a selected tea clone, 'TRI-2025', by temporary immersion., Plant Cell Rep., 19(12), 1140-1145.

[7] Akula, A., Dodd, W. A., 1998, Direct somatic embryogenesis in a selected tea clone, 'TRI-2025'(Camellia sinensis (L.) O. Kuntze) from nodal explants., Plant Cell Rep., 17(10), 804-809.

[8] Kato, M., 1986, Micropropagation through cotyledon culture in Camellia japonica L. and C. sinensis L., Jap. J. Breed., 36(1), 31-33. 
[9] Balasubramanian, S., Marimuthu, S., Rajkumar, R., Vinod Haridas., 2000, Somatic embryogenesis and multiple shoot induction in Camellia sinensis (L). O. Kuntze., J. Plantn. Crops., 28(1), 44-49.

[10] Xin, Y. LI., Feng, H. Huang., Brad Murphy, J., Edward, E., Gbur, J. R., 1998, Polyethylene glycol and maltose enhance somatic embryo maturation in Loplolly pine (Pinus taeda L.)., In vitro Cell Dev. Biol., 34(1), 22-26.

[11] Madakadze, R. M., and Senaratna, T., 2000, Effect of growth regulators on maturation of geranium (Pelargonium x hortorum) somatic embryos., Plant Growth Reg., 30(1), 55-60.

[12] Murashige, T., and Skoog, F., 1962, A revised medium for rapid growth and bioassays with tobacco tissue cultures., Physiol. Plant., 15, 473-497.

[13] Ravindra, B. Malabadi., Jaime, A. Teixeira da Silva., Gangadhar, S. Mulgund., 2011, Induction of somatic embryogenesis in Pinus caribaea., Tea and Forestry Sci. and Biotech., $5(1), 27-32$.

[14] Akula, A., Akula, C., Bateson, M., 2000, Betaine a novel candidate for rapid induction of somatic embryogenesis in tea (Camellia sinensis (L.) O. Kuntze)., Plant Growth Reg., 30(3), 241-246.

[15] Mithilesh, Singh., and Rakhi, Chaturvedi., 2009, An Efficient Protocol for Cyclic Somatic Embryogenesis in Neem (Azadirachta indica A Juss.)., Int. J. Civil and Env. Engg., 1(1), 49-51.

[16] Dusica, calic-Dragosavac., Snezana, Zdravkovi-Kora., 2010, Effect of activated charcoal, abscisic acid and polyethylene glycol on maturation, germination and conversion of Aescu- lus hippocastanum androgenic embryos., Afr. J. Biotech., 9(25), 3786-3793.

[17] Claudio, stasolla., and Edward, C. Yeung., 2003, Recent advances in conifer somatic embryogenesis: improving somatic embryo quality., Plant Cell Tissue and Organ Cult., 74(1), 15-35.

[18] Denchev, P., Velcheva, M., Atanassov, A., 1991, A new approach to direct somatic embryogenesis in Medicago., Plant Cell Rep., 10(6-7), 338-341.

[19] Langhansova, L., Konradova, H., Vanek, T., 2004, Polyethylene glycol and abscisic acid improve maturation and regeneration of Panax ginseng somatic embryos., Plant Cell Rep., 22(10), 725-730.

[20] Laurent, Linossier., Philippe, Veisseire., Françoise, Cailloux., Alain, Coudret., 1997, Effects of abscisic acid and high concentrations of PEG on Hevea brasiliensis somatic embryos development., Plant Sci., 124(2), 183-191.

[21] Attree, S. M., Pomeroy, M. K., Fowke, L. C., 1995, Development of white spruce (Picea glauca (Moench) Voss) somatic embryos during culture with abscisic acid and osmoticum, and their tolerance to drying and frozen storage., J. Exp. Bot., 46(4), 433-439.

[22] Misra, S., Attree, S. M., Leal, L., Fowke, L. C., 1993, Effect of abscisic acid, osmoticum, and desiccation on synthesis of storage proteins during the development of white spruce somatic embryos., Ann. Bot, 71(1), 11-22.

[23] Salunkhe, C. K., Rao, P. S., Mhatre, M., 1999, Plantlet regeneration via somatic embryogenesis in anther callus of vitis latifolia L., Plant Cell Rep., 8(7/8), 670-673. 